disease [3]. Inheritance of the APOE e4 genotype is an independent risk factor for developing higher levels of amyloid accumulation.

\title{
References.
}

1. McDonald J, Krainc D. JAMA. 2014 Jan 15;311(3):298-9.

2. Growdon JH, Hyman BT. JAMA Neurol. 2014 Jan 1;71(1):7-8.

3. Hyman BT, et al. Arch Neurol. 1995 Apr;52(4):373-8.

\section{RIBOFLAVIN IN BROWN-VIALETTO-VAN LAERE SYNDROME}

Investigators at Great Ormond Street Hospital, London, UK, and multiple centers internationally report the response to high-dose oral riboflavin therapy in 18 patients from 13 families with mutations in SLC5ZA2, encoding riboflavin transporter RTVT2, a new causative gene for Brown-Vialetto-Van Laere syndrome (BVVLS), a progressive neurodegenerative disorder leading to death in childhood. BVVLS is characterized by cranial neuropathies, pontobulbar palsy, sensorimotor neuropathy manifesting with sensory ataxia, weakness of upper limbs and axial muscles, with preserved strength of lower limbs, optic atrophy, sensorineural hearing loss, and respiratory insufficiency. Riboflavin therapy resulted in significant sustained clinical and biochemical improvement in 2 patients and preliminary response in 13 patients. (Foley AR, Menezes MP, Pandraud A, et al. Treatable childhood neuronopathy caused by mutations in riboflavin transporter RFVT2. Brain 2014 Jan;137(Pt 1):44-56).

COMMENTARY. BVVLS is a similar disorder to Fazio Londe syndrome caused by subtly different mutations of the same gene, and with the additional clinical feature of sensorineural deafness [1][2]. Diagnosis requires mutation analysis of transporter genes. The simple treatment with riboflavin supplementation may halt progression of both neurodegenerative disorders. An invited comment by Dr. John Wilson, Emeritus Chief of Neurology, Great Ormond Street Hospital, London, UK, and an authority on Fazio-Londe disease [2], is paraphrased as follows: "as our understanding of the basic concepts of disease become more complex, so we are lead to a beautiful simplicity (in the form of vitamin therapy) that brings light into dark places." How many similar degenerative diseases may in the future be found responsive to a simple vitamin?

References.

1. Bosch AM, et al. Orphanet J Rare Dis. 2012 Oct 29;7:83.

2. McShane MA, et al. Brain. 1992 Dec;115 ( Pt 6):1889-900.

\section{AUTISM SPECTRUM DISORDERS}

\section{ABNORMAL MOTOR FUNCTION AND AUTISM}

Investigators from Albert Einstein College of Medicine, Bronx, NY, recorded the gait characteristics and prevalence of toe walking, the range of passive joint mobility, and age at walking in children with DSM IV autism spectrum disorders (ASDs) and in ageand gender-matched healthy peers (mean age 4 years 6 months, range 22 months -10 
years 9 months). Children with ASD had significantly greater passive joint mobility, more gait abnormalities, and walked on average 1.6 months later than their non-autistic peers. Gait abnormalities included wide-based, apraxic, posturing, clumsy and toe walking. This study indicates that ASDs affect a broader range of central nervous system circuitry than often appreciated. (Klein MS, et al. Abnormalities of joint mobility and gait in children with autism spectrum disorders. Brain Dev 2014 Feb;36(2):91-6).

COMMENTARY. Motor abnormalities in addition to sociability, communication, and restricted and repetitive behaviors should be examined in the clinical evaluation of a child suspected of having autism spectrum disorder. Hypotonia is commonly recorded in the neurological examination of a child with ASD. The most reliable estimation of tone is the resistance to passive movement of a limb [1]. The authors emphasize that hypotonia has multiple potential causes, and in children with ASD the relative contribution of peripheral ligamentous joint laxity, muscle disorder or central nervous system dysfunction was not determined.

\section{References.}

1.Denny-Brown D. Handbook of Neurological Examination and Case Recording. Cambridge, MA: Harvard University Press; 1965.

\section{SLEEP PATTERNS AND AUTISM}

Investigators from University of Bristol, and other centers in the UK and Canada conducted a prospective study of sleep duration of children with an autism spectrum disorder (ASD) diagnosis at age 11 years $(n=73)$. Parental reports of sleep duration collected by questionnaires at 8 time points from 6 months to 11 years showed that from age 30 months to 11 years, children with ASD slept for 17-43 min less each day than contemporary controls. In infancy, no significant difference in total sleep duration was apparent, but from 30 months of age, children with ASD had less nocturnal sleep than their peers. Night-time sleep duration was shortened by later bedtimes, earlier waking times and frequent waking ( 3 or more times a night). Age specific decreases of $>1$ SD in sleep duration was a predictor of ASD between 18 months and 30 months of age $(p=0.04)$ and from 30 months to 42 months $(\mathrm{p}=0.02$ ). (Humphreys JS, Gringras P, Blair PS, et al. Sleep patterns in children with autistic spectrum disorders: a prospective cohort study. Arch Dis Child 2014 Feb;99(2):114-8).

COMMENTARY. Sleep duration in children with ASD is reduced from 30 months of age and persists until adolescence. Changes in sleep patterns are most noticeable between 18 months and 3 and one half years. Children with ASD are reported to have reduced levels of circulating melatonin and disrupted circadian rhythms [1]. Melatonin should be considered in the treatment of sleep patterns in ASD children [2].

References.

1. Rossignol DA, Frye RE. Dev Med Child Neurol. 2011 Sep;53(9):783-92.

2. Malow B, et al. J Autism Dev Disord. 2012 Aug;42(8):1729-37. 